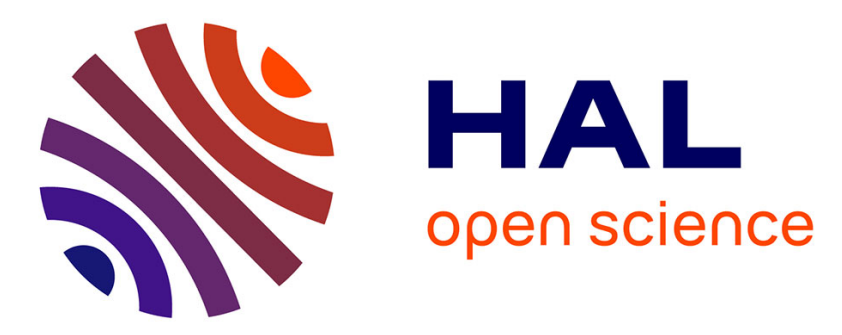

\title{
Analytic Variations on Redundancy Rates of Renewal Processes
}

Philippe Flajolet, Wojtek Szpankowski

\section{To cite this version:}

Philippe Flajolet, Wojtek Szpankowski. Analytic Variations on Redundancy Rates of Renewal Processes. [Research Report] RR-3553, INRIA. 1998. inria-00073130

\section{HAL Id: inria-00073130 \\ https://hal.inria.fr/inria-00073130}

Submitted on 24 May 2006

HAL is a multi-disciplinary open access archive for the deposit and dissemination of scientific research documents, whether they are published or not. The documents may come from teaching and research institutions in France or abroad, or from public or private research centers.
L'archive ouverte pluridisciplinaire HAL, est destinée au dépôt et à la diffusion de documents scientifiques de niveau recherche, publiés ou non, émanant des établissements d'enseignement et de recherche français ou étrangers, des laboratoires publics ou privés. 


\section{Analytic Variations on Redundancy Rates of Renewal Processes}

Philippe Flajolet, Wojtek Szpankowski

$\mathbf{N}^{\circ} \mathbf{3 5 5 3}$

Novembre 1998

THÈME 2 

Thème 2 - Génie logiciel et calcul symbolique Projet Algorithmes

Rapport de recherche- Novembre 1998 - 12 pages

\title{
Analytic Variations on Redundancy Rates of Renewal Processes
}

Philippe Flajolet, Wojtek Szpankowski

\begin{abstract}
Csiszár and Shields have recently proved that the minimax redundancy for a class of renewal processes is $\Theta(\sqrt{n})$ where $n$ is the block length. This interesting result provides a first non-trivial bound on redundancy for a non-parametric family of processes. The present paper provides a precise estimate up to the constant term of the redundancy rate for such sources. The asymptotic expansion is derived by complex-analytic methods that include generating function representations, Mellin transforms, singularity analysis and saddle point estimates. This work places itself within the framework of analytic information theory.
\end{abstract}

Keywords. Analytic information theory, redundancy, Mellin transform, saddle point method, singularity analysis. 


\section{Variations analytiques sur le taux de redondance des processus de renouvellement}

Résumé : Csiszár et Shields ont montré récemment que la redondance de la classe des processus de renouvellement est $\Theta(\sqrt{n})$, où $n$ représente la taille des blocs. Ce résultat intéressant fournit la première borne non triviale portant sur la redondance d'une famille non paramétrique de processus. Notre article fournit une évaluation précise de la redondance de telles sources, ce jusqu'au terme constant. Le développement asymptotique est assuré par des méthodes d'analyse asymptotique complexe incluant représentations par séries génératrices, transformation de Mellin, analyse de singularité et estimations de col. Ce travail se situe dans le cadre de la théorie analytique de l'information.

Mots clefs. Théorie analytique de l'information, redondance, transformation de Mellin, méthode de col, analyse de singularité. 


\title{
Analytic Variations on Redundancy Rates of Renewal Processes
}

\author{
Philippe Flajolet and Wojciech Szpankowski Senior Member, IEEE
}

\begin{abstract}
Csiszár and Shields have recently proved that the minimax redundancy for a class of renewal processes is $\Theta(\sqrt{n})$ where $n$ is the block length. This interesting result provides a first non-trivial bound on redundancy for a nonparametric family of processes. The present paper provides a precise estimate of the redundancy rate for such sources, namely,
\end{abstract}

$$
\frac{2}{\log 2} \sqrt{\left(\frac{\pi^{2}}{6}-1\right) n}-\frac{5}{8} \log _{2} n+\frac{1}{2} \log _{2} \log n+O(1) .
$$

This asymptotic expansion is derived by complex-analytic methods that include generating function representations, Mellin transforms, singularity analysis and saddle point estimates. This work places itself within the framework of analytic information theory.

Keywords - Redundancy, universal coding, renewal processes, partitions of integers, tree function, Mellin transform, saddle point method, analytic information theory.

\section{INTRODUCTION}

$\mathrm{R}$ ECENT YEARS have seen a resurgence of interest in redundancy rates of lossless coding; see [3], [13], [15], [17], [18], [19], [20], [22], [24]. The redundancy-rate problem of universal fixed-to-variable length coding for a class of sources consists in determining by how much the actual code length exceeds the optimal (ideal) code length. In a minimax scenario one finds an acceptable code such that the maximal redundancy over all sources within a certain class is as small as possible. While Shields [18] proved that there is no function $o(n)$ which is a rate bound on the redundancy for the class of all ergodic processes, it has been known for some time (cf. [15], [22]) that, for certain parametric families of sources (e.g., memoryless and Markov sources), the redundancy can be as small as $\Theta(\log n)$ where $n$ is the block length. There was no interesting bound for a class of sources that lies between $\Theta(\log n)$ and general $o(n)$ until recently, when Csiszár and Shields [3] designed a renewal class of sources that yields a $\Theta(\sqrt{n})$ bound. Still one would like to know more about this bound. What is for instance the constant in front of $\sqrt{n}$, if there is one? (See [13] for an example where fluctuations are involved in the redundancy rate.) Is the next term of the redundancy $100 \sqrt{n / \log n}$ or $2 \log n$ ? And so forth. In this paper, we address these questions by providing an asymptotic

P. Flajolet is with the Algorithms Project, INRIA-Rocquencourt F78153 Le Chesnay (France). Email: Philippe.Flajolet@inria.fr

W. Szpankowski is with the Department of Computer Science, Purdue University, W. Lafayette, IN 47907 (U.S.A.). Email: spa@cs . purdue.edu

The work of P. Flajolet was supported in part by the ESPRIT Basic Research Action No. 7141 (ALCOM II). The work of W. Szpankowski was supported by NSF Grants NCR-9415491 and C-CR-9804760, and NATO Collaborative Grant CRG.950060. expansion of the redundancy for renewal sources up to the constant term.

Regarding coding theory, we shall follow the notation and the presentation from [3]. A code $C_{n}: \mathcal{A}^{n} \rightarrow\{0,1\}^{*}$ is defined as a mapping from the set $\mathcal{A}^{n}$ of all sequences of length $n$ over the alphabet $\mathcal{A}$ to the set $\{0,1\}^{*}$ of binary sequences. A message of length $n$ with letters indexed from 1 to $n$ is denoted by $x_{1}^{n}$, so that $x_{1}^{n} \in \mathcal{A}^{n}$. We write $X_{1}^{n}$ to denote the random variable representing a message of length $n$. Given a probabilistic source model, we let $P\left(x_{1}^{n}\right)$ be the probability of the message $x_{1}^{n}$; given a code $C_{n}$, we let $L\left(C_{n}\right)$ be the code length. Information-theoretic quantities are expressed in binary logarithms written $\lg :=\log _{2}$, but sometimes we also use $\log :=\ln$.

From Shannon's works we know that the entropy $H_{n}(P)=-\sum_{x_{1}^{n}} P\left(x_{1}^{n}\right) \lg P\left(x_{1}^{n}\right)$ is an absolute lower bound on the expected code length. Hence $-\lg P\left(x_{1}^{n}\right)$ can be viewed as the "ideal" code length. (Actually, the ShannonFano code of length $\left[-\lg P\left(x_{1}^{n}\right)\right\rceil$ attains the lower bound $H_{n}(P)$ within one bit.) The next natural question is to ask by how much the length $L\left(C_{n}\right)$ of a code differs from the ideal code length, either for individual sequences or on average. Thus, the pointwise redundancy $R_{n}\left(C_{n}, P ; x_{1}^{n}\right)$ and the average redundancy $\bar{R}_{n}\left(C_{n}, P\right)$ are defined as

$$
\begin{aligned}
R_{n}\left(C_{n}, P ; x_{1}^{n}\right) & =L\left(C_{n}\right)+\lg P\left(x_{1}^{n}\right) \\
\bar{R}_{n}\left(C_{n}\right) & =\mathbf{E}_{X_{1}^{n}}\left[R_{n}\left(C_{n}, P ; X_{1}^{n}\right)\right] \\
& =\mathbf{E}\left[L\left(C_{n}\right)\right]-H_{n}(P)
\end{aligned}
$$

where the underlying probability measure $P$ represents a particular source model and $\mathbf{E}$ denotes the expectation. Another natural measure of code performance is the maximal redundancy defined as

$$
R^{*}\left(C_{n}, P\right)=\max _{x_{1}^{n}}\left\{R_{n}\left(C_{n}, P ; x_{1}^{n}\right)\right\} .
$$

Observe that while the pointwise redundancy can be negative, maximal and average redundancies cannot, by Shannon's theory.

In practice, the source probabilities are unknown, hence the desire to design codes for a whole class of source models $\mathcal{S}$. When the source is known, the redundancy can be as low as 1 bit, as demonstrated by Shannon-Fano codes. Therefore, for unknown probabilities, the redundancy rate can be also viewed as the penalty paid for estimating the underlying probability measure. More precisely, universal codes are those for which the redundancy is $o(n)$ for all $P \in \mathcal{S}$. The weak redundancy-rate problem for the class 
$\mathcal{S}$ can be roughly viewed as finding a bound on the redundancy rate for a sequence of codes $C_{n}$ over all $P \in \mathcal{S}$ (cf. [3]). The (asymptotic) strong redundancy-rate problem consists in determining for a class $\mathcal{S}$ of source models the rate of growth of the minimax quantities

$$
\begin{aligned}
\bar{R}_{n}^{*}(\mathcal{S}) & =\min _{C_{n}} \max _{P \in \mathcal{S}}\left\{\bar{R}_{n}\left(C_{n}, P\right)\right\}, \\
R_{n}^{*}(\mathcal{S}) & =\min _{C_{n}} \max _{P \in \mathcal{S}}\left\{R_{n}^{*}\left(C_{n}, P\right)\right\}
\end{aligned}
$$

as $n \rightarrow \infty$. In this paper, we deal with the stronger version, namely with the minimax redundancy $R_{n}^{*}$ for arbitrary renewal sources.

The redundancy rate problem is typical of a situation where second-order asymptotics play a crucial rôle since the leading term of $L\left(C_{n}\right)$ is known to be $n H$, where $H$ is the entropy rate. This problem is an ideal candidate for "analytic information theory" that applies analytic tools to information theory. As argued by Andrew Odlyzko [14] "Analytic methods are extremely powerful and when they apply, they often yield estimates of unparalleled precision." We shall see this principle at work for the redundancy problem. (Other examples are provided by [10], [11], [13], [20], [21].) In fact, in his 1997 Shannon Lecture [25], Jacob Ziv presented compelling arguments for "backing off" to a certain degree from the (first-order) asymptotic analysis of information systems in order to predict the behaviour of real systems where we always face finite (and often small) lengths (of sequences, files, codes, etc.) One way of overcoming these difficulties is to increase the accuracy of asymptotic analysis and replace first-order analyses (e.g., a leading term of the average code length) by more complete asymptotic expansions, thereby extending their range of applicability to smaller values while providing more accurate analyses (like constructive error bounds, large deviations, local or central limit laws).

A substantial literature is available on the redundancy problem. The following results are known.

- If $\mathcal{M}$ is i.i.d. or the class of Markov chains, or more generally the process belongs to a finitely parametrizable class of dimension $K$, then Rissanen [15] established

$$
\bar{R}_{n}(\mathcal{M}) \sim R_{n}^{*}(\mathcal{M}) \sim \frac{K}{2} \lg n .
$$

It was also found in [22] that the next term of $\bar{R}_{n}(\mathcal{S})$ and of $R_{n}^{*}(\mathcal{S})$ is $O(1)$. In fact, Szpankowski [20] has established a full asymptotic expansion for $R_{n}^{*}(\mathcal{S})$ for memoryless sources over an $m$-ary alphabet, namely

$$
R_{n}^{*}(\mathcal{M})=\frac{m-1}{2} \lg \left(\frac{n}{2}\right)+\lg \left(\frac{\sqrt{\pi}}{\Gamma(m / 2)}\right)+\cdots,
$$

where $\Gamma(x)$ is the Euler gamma function. In passing we observe that when the alphabet size $m$ is large, the second order terms may contribute significantly to $R_{n}^{*}$. More importantly, the above formula is true only when $m$ is fixed, while in some applications $m$ may depend on $n$ (e.g., image size is comparable to image alphabet). Then, one needs a uniform asymptotic expansion of $R_{n}^{*}$, and clearly second order terms will contribute to the final outcome.

- Csiszàr and Shields [3] have studied order $r$ Markov renewal sequences in which a 1 is inserted every $T_{0}, T_{1}, \ldots$ of 0 's, where $\left\{T_{i}\right\}$ is either an i.i.d. or Markov renewal or $r$-order Markov renewal process. We denote such sources as $\mathcal{R}_{r}$. The authors of [3] proved that $\bar{R}_{n}\left(\mathcal{R}_{r}\right)=R^{*}\left(\mathcal{R}_{r}\right)=$ $\Theta\left(n^{(r+1) /(r+2}\right)$ for $r=1,2, \ldots$ which specializes to $\Theta(\sqrt{n})$ when $r=0$.

- Shields [18] proved that there is no function $\rho(n)=o(n)$ which is a weak-rate bound for the class of all ergodic processes.

- Louchard and Szpankowski [13], Savari [17], Wyner [24], and Jacquet and Szpankowski [12] proved that the LempelZiv codes in the class of i.i.d. and Markov processes have either rate $\Theta(n / \log n)$ (for LZ'78) or $\Theta(n \log \log n / \log n)$ (for LZ'77 code). Interestingly, in [13] it was shown that for LZ'78 the bound $\Theta(n / \log n)$ cannot be improved to an asymptotic equivalence since a fluctuating function is involved. More precisely, for a binary alphabet with 0's generated with probability $p$ and 1's with probability $q=$ $1-p$, the authors of [13] showed that

$$
\bar{R}_{n}(L Z)=(\chi+\delta(n)) \frac{n}{\log n}+O\left(\frac{n \log \log n}{\log ^{2} n}\right),
$$

where $\chi$ is an explicitly determined constant. What is more surprising is the occurrence of the function $\delta(x)$ that fluctuates with mean zero and a tiny amplitude for $\log p / \log q$ rational (the amplitude of $\delta(x)$ is smaller than $10^{-6}$ for the unbiased case, where $p=q=0.5$ ), but satisfies $\lim _{x \rightarrow \infty} \delta(x)=0$ otherwise.

In this paper, we revisit Csiszàr and Shields renewal process and present a precise analysis of the redundancy rate for the class of basic renewal source $\mathcal{R}_{0}$ corresponding to $r=0$ (see Section II for details). Our main result is as follows.

Theorem 1: Consider the class $\mathcal{R}_{0}$ of renewal sources. Let $c=\frac{\pi^{2}}{6}-1 \approx 0.645$. Then the minimax redundancy $R_{n}^{*}\left(\mathcal{R}_{0}\right)$ satisfies, for large $n$,

$$
R_{n}^{*}\left(\mathcal{R}_{0}\right)=R_{n}+\frac{1}{2}+\eta_{n}
$$

with $\eta_{n} \in\left[-\frac{1}{2}, \frac{1}{2}\right]$ and

$$
R_{n}=\frac{2}{\log 2} \sqrt{c n}-\frac{5}{8} \lg n+\frac{1}{2} \lg \log n+\frac{1}{8} \lg \frac{c}{2^{12} \pi^{2}}+o(1)
$$

where $\lg :=\log _{2}$.

This paper is organized as follows. In Section II, the problem is reduced to estimating a certain combinatorial sum that is of independent interest (Lemma 1). Next, we present the main ingredients of the proof in Section III. The heart of the analysis is Lemma 2 that is established in Section IV. The proof is analytic and uses such diverse tools as the Mellin transform, singularity analysis, and the 
saddle point method. We believe that the analytic approach discussed in this section is of general interest and hope it may find further applications in information theory. For this reason as well as for convenience of exposition, we adopt a tabular presentation of the two main tools used here: the Mellin transform (Fig. 1) and the saddle point method (Fig. 2).

\section{Reduction to a Combinatorial Sum}

We start with a precise definition of the class $\mathcal{R}_{0}$ of renewal process and its associated sources. Let $T_{1}, T_{2} \ldots$ be a sequence of i.i.d. positive-valued random variables with distribution $Q(j)=\operatorname{Pr}\left\{T_{1}=j\right\}$. An independent random variable $T_{0}$ is introduced with distribution

$$
\operatorname{Pr}\left\{T_{0}=i\right\}=\mathbf{E}\left[T_{1}\right]^{-1} \sum_{j \geq i} Q(j)
$$

provided $\mathbf{E}\left[T_{1}\right]<\infty$. The quantities $\left\{T_{i}\right\}_{i=1}^{\infty}$ are the interarrival times, while $T_{0}$ is the initial waiting time. The process $T_{0}, T_{0}+T_{1}, T_{0}+T_{1}+T_{2}, \ldots$ is then called a renewal process and it is stationary whenever $T_{0}$ has the distribution above. With such a renewal process there is associated a binary renewal sequence that is a $0,1-$ sequence in which the 1's occur exactly at the renewal epochs $T_{0}, T_{0}+T_{1}, T_{0}+T_{1}+T_{2}$, etc. Since $P$ and $Q$ determine one another, we often identify the underlying stationary probability measure $P$ defined on $\{0,1\}^{\infty}$ with the distribution $Q$ of the interarrival times that it induces.

We now briefly discuss the mathematical aspect of the redundancy problem. Let $x_{1}^{n}$ be a renewal sequence and $L\left(C_{n}, x_{1}^{n}\right)$ a code assigned to it. Our goal is to derive asymptotics of

$$
R_{n}^{*}(\mathcal{S})=\min _{C_{n}} \sup _{Q \in \mathcal{S}} \max _{x_{1}^{n}}\left\{L\left(C_{n}, x_{1}^{n}\right)+\lg P\left(x_{1}^{n}\right)\right\}
$$

where the supremum is taken over all distributions $Q$. Shtarkov's maximum-likelihood technique [19] implies

$$
\lg \left(\sum_{x_{1}^{n}} \sup _{Q} P\left(x_{1}^{n}\right)\right) \leq R_{n}^{*}(\mathcal{S}) \leq \lg \left(\sum_{x_{1}^{n}} \sup _{Q} P\left(x_{1}^{n}\right)\right)+1
$$

Indeed, for the lower bound Shtarkov [19] observed that

$$
q\left(x_{1}^{n}\right):=\frac{\sup _{Q} P\left(x_{1}^{n}\right)}{\sum_{x_{1}^{n}} \sup _{Q} P\left(x_{1}^{n}\right)}
$$

is a probability distribution. Hence, by Kraft's inequality there exists $\widetilde{x}_{1}^{n}$ such that (for uniquely decodable codes $C_{n}$ )

$$
-L\left(C_{n}\right) \leq \lg q\left(\widetilde{x}_{1}^{n}\right)
$$

which implies the lower bound. For the upper bound, Shtarkov proposed a code $C_{n}$ of length

$$
L\left(\widetilde{C}_{n}\right)=\left\lceil\lg \left(\sum_{x_{1}^{n}} \sup _{Q} P\left(x_{1}^{n}\right)\right)-\lg P\left(x_{1}^{n}\right)\right\rceil
$$

which gives the desired upper bound.

Let now $R_{n}$ be defined by

$$
R_{n}:=\lg \left(\sum_{x_{1}^{n}} \sup _{Q} P\left(x_{1}^{n}\right)\right) .
$$

The inequalities (2) mean that $R_{n} \leq R_{n}^{*}(\mathcal{S}) \leq R_{n}+1$, so that $R_{n}^{*}(\mathcal{S})$ is at distance at most $\frac{1}{2}$ from the middle of the interval $R_{n}+\frac{1}{2}$. This is precisely what is expressed by Equation (1) and the condition $\eta_{n} \in\left[-\frac{1}{2}, \frac{1}{2}\right]$.

We shall study instead of $R_{n}$ a closely related quantity, namely

$$
r_{n}=2^{R_{n}}
$$

It turns out that the problem of estimating $r_{n}$, hence $R_{n}$ and $R_{n}^{*}\left(\mathcal{R}_{0}\right)$, reduces to the evaluation of a purely combinatorial sum. (By convention here $0^{0}=1$.)

Lemma 1: The quantity $r_{n}=2^{R_{n}}$ admits the combinatorial form

$$
\left\{\begin{aligned}
r_{n} & =\sum_{k=0}^{n} r_{n, k} \\
r_{n, k} & =\sum_{\mathcal{P}(n, k)}\left(\begin{array}{c}
k \\
k_{0} \cdots k_{n-1}
\end{array}\right)\left(\frac{k_{0}}{k}\right)^{k_{0}} \cdots\left(\frac{k_{n-1}}{k}\right)^{k_{n-1}}
\end{aligned}\right.
$$

where $\mathcal{P}(n, k)$ denotes the set of partitions of $n$ into $k$ summands, that is, the collection of tuples of nonnegative integers $\left(k_{0}, k_{1}, \ldots, k_{n-1}\right)$ satisfying

$$
\begin{aligned}
n & =k_{0}+2 k_{1}+\cdots+n k_{n-1}, \\
k & =k_{0}+k_{1}+\cdots+k_{n-1} .
\end{aligned}
$$

Proof: As a first step in investigating (3), we address the problem of finding the supremum of $P\left(x_{1}^{n}\right)$ over all distributions $Q \in \mathcal{R}_{0}$, that is, we solve the optimization problem $\sup _{Q} P\left(x_{1}^{n}\right)$.

Observe that the renewal sequence $x_{1}^{n}$ can be represented as

$$
x_{1}^{n}=0^{\alpha_{1}} 10^{\alpha_{2}} 1 \cdots 10^{\alpha_{n}} 1 \underbrace{0 \cdots 0}_{k^{*}}
$$

where $0 \leq \alpha_{i} \leq n$ for $i=1, \ldots, n$. Let $k_{m}$ be the number of $i$ such that $\alpha_{i}=m$, where $m=0,1, \ldots, n-1$. Then

$$
P\left(x_{1}^{n}\right)=Q^{k_{0}}(0) Q^{k_{1}}(1) \cdots Q^{k_{n-1}}(n-1) Q^{*}\left(k^{*}\right)
$$

subject to $Q(0)+Q(1)+\cdots+Q(n-1) \leq 1$, where

$$
k_{0}+2 k_{1}+\cdots+n k_{n-1}+k^{*}=n,
$$

and $Q^{*}\left(k^{*}\right)=\operatorname{Pr}\left\{T_{1} \geq k^{*}\right\}$.

What is now needed is the supremum of $P\left(x_{1}^{n}\right)$ represented by (7) over all distributions $Q(\cdot)$. But this is a simple optimization problem with inequality constraints. By the Kuhn-Tucker condition or otherwise, it is seen that the values $Q^{*}\left(k^{*}\right)=1$ and

$$
\frac{Q(i)}{Q(j)}=\frac{k_{i}}{k_{j}} \quad \text { for } 1 \leq i, j \leq n
$$


optimize $P\left(x_{1}^{n}\right)$. In view of the above, we conclude that $\sup _{Q} P\left(x_{1}^{n}\right)=\left(\frac{k_{0}}{k_{0}+\cdots+k_{n-1}}\right)^{k_{0}} \cdots\left(\frac{k_{n-1}}{k_{0}+\cdots+k_{n-1}}\right)^{k_{n-1}}$

where $n$ and $k$ are constrained by (5) and (6). These last two conditions represent an arbitrary partition of the integer $n$ into $k$ summands.

It can also be observed that the quantity $r_{n}$ has an intrinsic meaning. Let $\mathcal{W}_{n}$ denote the set of all $n^{n}$ sequences of length $n$ over the alphabet $\{0, \ldots, n-1\}$. For a sequence $w$, take $k_{j}$ to be the number of letters $j$ in $w$. Then each sequence $w$ carries a "maximum likelihood probability", $\pi_{M L}(w)$, given by the right hand-side of (9): this is the probability that $w$ gets assigned in the Bernoulli model that makes it most likely. The quantity $r_{n}$ is also

$$
r_{n}=\sum_{w \in \mathcal{W}_{n}} \pi_{M L}(w)
$$

\section{A Streamlined Analysis}

Our goal is to estimate asymptotically $r_{n}$ through asymptotics of $r_{n, k}$. A difficulty of finding such asymptotics stems from the factor $k ! / k^{k}$ present in the definition (4) of $r_{n, k}$. We circumvent this problem by analyzing a related pair of sequences, namely $s_{n}$ and $s_{n, k}$ that are defined as

$$
\left\{\begin{array}{c}
s_{n}= \\
\sum_{k=0}^{n} s_{n, k} \\
s_{n, k}=e^{-k} \sum_{\mathcal{P}(n, k)} \frac{k^{k_{0}}}{k_{0} !} \cdots \frac{k^{k_{n-1}}}{k_{n-1} !} .
\end{array}\right.
$$

The translation from $s_{n}$ to $r_{n}$ is most conveniently expressed in probabilistic terms. Introduce the random variable $K_{n}$ whose probability distribution is $s_{n, k} / s_{n}$, that is,

$$
\varpi_{n}: \quad \operatorname{Pr}\left\{K_{n}=k\right\}=\frac{s_{n, k}}{s_{n}},
$$

where $\varpi_{n}$ denotes the distribution. Then Stirling's formula yields

$$
\begin{aligned}
\frac{r_{n}}{s_{n}} & =\sum_{k=0}^{n} \frac{r_{n, k}}{s_{n, k}} \frac{s_{n, k}}{s_{n}}=\mathbf{E}\left[\left(K_{n}\right) ! K_{n}^{-K_{n}} e^{-K_{n}}\right] \\
& =\mathbf{E}\left[\sqrt{2 \pi K_{n}}\right]+O\left(\mathbf{E}\left[K_{n}^{-\frac{1}{2}}\right]\right) .
\end{aligned}
$$

Thus, the problem of finding $r_{n}$ reduces to asymptotic evaluations of $s_{n}, \mathbf{E}\left[\sqrt{K_{n}}\right]$ and $\mathbf{E}\left[K_{n}^{-\frac{1}{2}}\right]$. The heart of the matter is the following lemma which provides the necessary estimates.

Lemma 2: Let $\mu_{n}=\mathbf{E}\left[K_{n}\right]$ and $\sigma_{n}^{2}=\operatorname{Var}\left(K_{n}\right)$, where $K_{n}$ has the distribution $\varpi_{n}$ defined above in (11). The following holds

$$
\begin{aligned}
s_{n} & \sim \exp \left(2 \sqrt{c n}-\frac{7}{8} \log n+d+o(1)\right) \\
\mu_{n} & =\frac{1}{4} \sqrt{\frac{n}{c}} \log \frac{n}{c}+o(\sqrt{n}) \\
\sigma_{n}^{2} & =O(n \log n)=o\left(\mu_{n}^{2}\right),
\end{aligned}
$$

where $c=\pi^{2} / 6-1, d=-\log 2-\frac{3}{8} \log c-\frac{3}{4} \log \pi$.

The somewhat delicate proof of Lemma 2 constitutes the core of the paper and it is deferred till the next section. Once the estimates of Lemma 2 are granted, the moments of order $\pm \frac{1}{2}$ of $K_{n}$ follow by a standard argument based on concentration of the distribution $\varpi_{n}$.

Lemma 3: For large $n$

$$
\begin{aligned}
\mathbf{E}\left[\sqrt{K_{n}}\right] & =\mu_{n}^{1 / 2}(1+o(1)) \\
\mathbf{E}\left[K_{n}^{-\frac{1}{2}}\right] & =o(1) .
\end{aligned}
$$

where $\mu_{n}=\mathbf{E}\left[K_{n}\right]$.

Proof: We only prove (16) since (17) is obtained in a similar manner. The upper bound $\mathbf{E}\left[\sqrt{K_{n}}\right] \leq \sqrt{\mathbf{E}\left[K_{n}\right]}$ follows from concavity of the function $\sqrt{x}$. The lower bound follows from concentration of the distribution. Chebyshev's inequality and (15) of Lemma 2 entail, for any arbitrarily small $\varepsilon>0$,

$$
\operatorname{Pr}\left\{\left|K_{n}-\mu_{n}\right|>\varepsilon \mu_{n}\right\} \leq \frac{\operatorname{Var}\left[K_{n}\right]}{\varepsilon^{2} \mu_{n}^{2}}=\frac{\delta(n)}{\varepsilon^{2}}
$$

where $\delta(n) \rightarrow 0$ as $n \rightarrow \infty$. Then

$$
\begin{aligned}
\mathbf{E}\left[\sqrt{K_{n}}\right] & \geq \sum_{k \geq(1-\varepsilon) \mu_{n}} \sqrt{k} \operatorname{Pr}\left\{K_{n} \geq k\right\} \\
& \geq(1-\varepsilon)^{\frac{1}{2}} \mu_{n}^{1 / 2} \operatorname{Pr}\left\{K_{n} \geq(1-\varepsilon) \mu_{n}\right\} \\
& \geq(1-\varepsilon)^{\frac{1}{2}}\left(1-\frac{\delta(n)}{\varepsilon^{2}}\right) \mu_{n}^{1 / 2}
\end{aligned}
$$

Hence for any $\eta>0$ one has $\mathbf{E}\left[\sqrt{K_{n}}\right]>\mu_{n}^{1 / 2}(1-\eta)$ provided $n$ is large enough. This completes the proof.

In summary, $r_{n}$ and $s_{n}$ are related by

$$
\begin{aligned}
r_{n} & =s_{n} \mathbf{E}\left[\sqrt{2 \pi K_{n}}\right](1+o(1)) \\
& =s_{n} \sqrt{2 \pi \mu_{n}}(1+o(1)),
\end{aligned}
$$

by virtue of (12) and Lemma 3. This leads to

$$
R_{n}=\lg r_{n}=\lg s_{n}+\frac{1}{2} \lg \mu_{n}+\lg \sqrt{2 \pi}+o(1)
$$

At this point it suffices to apply the estimates provided by Lemma 2 in order to get $R_{n}$ up to $o(1)$ term. The proof of Theorem 1 is in turn complete since $\left|R_{n}^{*}\left(\mathcal{R}_{0}\right)-R_{n}-\frac{1}{2}\right| \leq \frac{1}{2}$, by (2) and (3).

\section{Complex Asymptotic Analysis}

This section provides precise asymptotic estimates for the quantity $s_{n}$ and for moments of the distribution $\varpi_{n}$ as expressed in (14) and (15) of Lemma 2. It turns out that the quantities $s_{n, k}$ and $s_{n}$ have generating functions $S(z, u)$ and $S(z, 1)$, respectively, that are infinite products involving the tree function of combinatorial analysis. The corresponding coefficient asymptotics are dictated by the behaviour at the singularity of greatest weight [5] - in the case at hand, the positive real singularity $z=1$ - so that we start 
by investigating asymptotics of $S(z, 1)$ as $z \rightarrow 1$. This itself requires a dedicated analysis by mean of the Mellin transform. Once the dominant singular behaviour of $S(z, 1)$ near $z=1$ has been found, it remains to extract information on the coefficients $s_{n}$. This task involves an appeal to the saddle point method (summarized by Lemma 4) and necessitates some technical concentration condition (Lemma 5). (The whole analysis draws its inspiration from a method of Meinardus in the asymptotic theory of integer partitions; see especially Chapter 6 of [1].) Proceeding in this way the estimate (13) of $s_{n}$ in Lemma 2 is established. Finally, the method adapts gracefully to moment estimates, yielding the other two estimates (14), (15) of Lemma 2.

Generating Functions. The expression of $s_{n, k}$ in (10) involves quantities of the form $k^{k} / k$ !. We start by introducing the well-known "tree function" $T(z)$ defined as the solution of

$$
T(z)=z e^{T(z)}
$$

that is analytic at 0 . The function $T(z)$ satisfies, by the Lagrange inversion theorem,

$$
T(z)=\sum_{k=1}^{\infty} \frac{k^{k-1}}{k !} z^{k}
$$

The tree function owes its name to its rôle in tree enumerations and we refer to the survey paper [2] for algebraic and analytic properties of this important special function of combinatorics.

Next define the function $\beta(z)$ as

$$
\beta(z)=\sum_{k=0}^{\infty} \frac{k^{k}}{k !} e^{-k} z^{k}
$$

One has (e.g., by Lagrange inversion again or otherwise)

$$
\beta(z)=\frac{1}{1-T\left(z e^{-1}\right)} .
$$

The quantities $s_{n}$ and $s_{n, k}$ of (10) have generating functions,

$$
S_{n}(u)=\sum_{k=0}^{\infty} s_{n, k} u^{k}, \quad S(z, u)=\sum_{n=0}^{\infty} S_{n}(u) z^{n} .
$$

Then, since equation (19) involves convolutions of sequences of the form $k^{k} / k$ !, we have

$$
\begin{aligned}
S(z, u) & =\sum_{\mathcal{P}_{n, k}} z^{1 k_{0}+2 k_{1}+\cdots}\left(\frac{u}{e}\right)^{k_{0}+\cdots+k_{n-1}} \frac{k^{k_{0}}}{k_{0} !} \cdots \frac{k^{k_{n-1}}}{k_{n-1} !} \\
& =\prod_{i=1}^{\infty} \beta\left(z^{i} u\right),
\end{aligned}
$$

We also need access to the first moment $\mu_{n}=\mathbf{E}\left[K_{n}\right]$ and the second factorial moment $\mathbf{E}\left[K_{n}\left(K_{n}-1\right)\right]$. They are obtained as

$$
\begin{aligned}
s_{n} & =\left[z^{n}\right] S(z, 1), \\
\mu_{n} & =\frac{\left[z^{n}\right] S_{u}^{\prime}(z, 1)}{\left[z^{n}\right] S(z, 1)} \\
\mathbf{E}\left[K_{n}\left(K_{n}-1\right)\right] & =\frac{\left[z^{n}\right] S_{u u}^{\prime \prime}(z, 1)}{\left[z^{n}\right] S(z, 1)}
\end{aligned}
$$

where $\left[z^{n}\right] F(z)$ denotes the coefficient at $z^{n}$ of $F(z)$, $S_{u}^{\prime}(z, 1)$ and $S_{u u}^{\prime \prime}(z, 1)$ represent the first and the second derivative of $S(z, u)$ at $u=1$.

Mellin Asymptotics. We deal here with $s_{n}$ that is accessible through its generating function,

$$
S(z, 1)=\prod_{i=1}^{\infty} \beta\left(z^{i}\right)
$$

The behaviour of the generating function $S(z, 1)$ as $z \rightarrow 1$ is an essential ingredient of the analysis.

First, the singularity of the tree function $T(z)$ at $z=e^{-1}$ is of the square-root type; see [2]. (This results from the failure of the implicit function theorem at $(z, T)=\left(e^{-1}, 1\right)$ where the relation becomes locally quadratic in $T$.) Hence, near $z=1, \beta(z)$ admits the singular expansion (cf. [2]):

$$
\beta(z)=\frac{1}{\sqrt{2(1-z)}}+\frac{1}{3}-\frac{\sqrt{2}}{24} \sqrt{(1-z)}++O(1-z) .
$$

We now turn to the infinite product asymptotics as $z \rightarrow$ $1^{-}$, with $z$ real. Let $L(z)=\log S(z, 1)$ and $z=e^{-t}$, so that

$$
L\left(e^{-t}\right)=\sum_{k=1}^{\infty} \log \beta\left(e^{-k t}\right) .
$$

Mellin transform techniques provide an expansion of $L\left(e^{-t}\right)$ around $t=0$ (or equivalently $z=1$ ) since the sum (24) falls under the harmonic sum paradigm of [6]. The Mellin approach is by now a standard technique in the analysis of algorithms. For the reader's convenience, we recall its main properties in Fig. 1, following [6], to which we refer globally for detailed validity conditions.

First the Mellin transform $L^{*}(s)=\mathcal{M}\left(L\left(e^{-t}\right) ; s\right)$ of $L\left(e^{-t}\right)$ is computed by the harmonic sum property (M3). For $\Re(s) \in(1, \infty)$, the transform evaluates to

$$
L^{*}(s)=\zeta(s) \Lambda(s)
$$

where $\zeta(s)=\sum_{n \geq 1} n^{-s}$ is the Riemann zeta function, and

$$
\Lambda(s)=\int_{0}^{\infty} \log \beta\left(e^{-t}\right) t^{s-1} d t .
$$

The subsequent treatment is typical of the Mellin analysis of harmonic sums: the singularity structure of $\Lambda(s)$ is deduced from the asymptotic properties of $\beta(z)$. This gives in turn the singularity structure of $L^{*}(s)$ that is then converted back into an asymptotic $\exp \left[\right.$ ansion of $L\left(e^{-t}\right)$. In 
(M1) Direct and Inverse Mellin Transforms. Let $c$ belong to the fundamental strip defined below.

$$
f^{*}(s):=\mathcal{M}(f(x) ; s)=\int_{0}^{\infty} f(x) x^{s-1} d x \quad \Longleftrightarrow \quad f(x)=\frac{1}{2 \pi i} \int_{c-i \infty}^{c+i \infty} f^{*}(s) x^{-s} d s .
$$

(M2) Fundamental Strip. The Mellin transform of $f(x)$ exists in the fundamental strip $\Re(s) \in(-\alpha,-\beta)$, where

$$
f(x)=O\left(x^{\alpha}\right) \quad(x \rightarrow 0), \quad f(x)=O\left(x^{\beta}\right) \quad(x \rightarrow \infty) .
$$

(M3) Harmonic Sum Property. By linearity and the scale rule $\mathcal{M}(f(a x) ; s)=a^{-s} \mathcal{M}(f(x) ; s)$,

$$
f(x)=\sum_{k \geq 0} \lambda_{k} g\left(\mu_{k} x\right) \quad \Longleftrightarrow \quad f^{*}(s)=g^{*}(s) \sum_{k \geq 0} \lambda_{k} \mu_{k}^{-s} .
$$

(M4) Mapping Properties (Asymptotic expansion of $f(x)$ and singularities of $f^{*}(s)$ ).

$$
f(x)=\sum_{(\xi, k) \in A} c_{\xi, k} x^{\xi}(\log x)^{k}+O\left(x^{M}\right) \quad \Longleftrightarrow \quad f^{*}(s) \asymp \sum_{(\xi, k) \in A} c_{\xi, k} \frac{(-1)^{k} k !}{(s+\xi)^{k+1}} .
$$

- (i) Direct Mapping. Assume that $f(x)$ admits as $x \rightarrow 0^{+}$the asymptotic expansion (22) for some $-M<-\alpha$ and $k>0$. Then for $\Re(s) \in(-M,-\beta)$, the transform $f^{*}(s)$ satisfies the singular expansion (22)

- (ii) Converse Mapping. Assume that $f^{*}(s)=O\left(|s|^{-r}\right)$ with $r>1$, as $|s| \rightarrow \infty$ and that $f^{*}(s)$ admits the singular expansion (22) for $\Re(s) \in(-M,-\alpha)$. Then $f(x)$ satisfies the asymptotic expansion (22) at $x=0^{+}$.

Fig. 1. Main properties of the Mellin transform.

effect, by the direct mapping property (M4), the expansion of $\beta(z)$ at $z=1$ implies

$$
\log \beta\left(e^{-t}\right)=-\frac{1}{2} \log t-\frac{1}{2} \log 2+O(\sqrt{t}),
$$

so that, collecting local expansions,

$$
\Lambda(s) \asymp(\Lambda(1))_{s=1}+\left(\frac{1}{2} \frac{1}{s^{2}}-\frac{1}{2} \frac{\log 2}{s}\right)_{s=0} .
$$

On the other hand, classical expansions give

$$
\zeta(s) \asymp\left(\frac{1}{s-1}+\gamma\right)_{s=1}+\left(-\frac{1}{2}-s \log \sqrt{2 \pi}\right)_{s=0} .
$$

Term-wise multiplication then provides the singular expansion of $L^{*}(s)$ :

$$
L^{*}(s) \asymp\left(\frac{\Lambda(1)}{s-1}\right)_{s=1}+\left(-\frac{1}{4 s^{2}}-\frac{\log \pi}{4 s}\right)_{s=0} .
$$

An application of the converse mapping property (M4) allows us to come back to the original function,

$$
L\left(e^{-t}\right)=\frac{\Lambda(1)}{t}+\frac{1}{4} \log t-\frac{1}{4} \log \pi+O(\sqrt{t}),
$$

which translates in

$L(z)=\frac{\Lambda(1)}{1-z}+\frac{1}{4} \log (1-z)-\frac{1}{4} \log \pi-\frac{1}{2} \Lambda(1)+O(\sqrt{1-z})$.
This computation is finally completed by the evaluation of $c:=\Lambda(1)$ :

$$
\begin{aligned}
c=\Lambda(1) & =-\int_{0}^{1} \log (1-T(x / e)) \frac{d x}{x} \\
& =-\int_{0}^{1} \log (1-t) \frac{(1-t)}{t} d t \quad\left(x=t e^{1-t}\right) \\
& =\frac{\pi^{2}}{6}-1 .
\end{aligned}
$$

In summary, we just proved that, as $z \rightarrow 1^{-}$,

$$
S(z, 1)=e^{L(z)}=a(1-z)^{\frac{1}{4}} \exp \left(\frac{c}{1-z}\right)(1+o(1)),
$$

where $a=\exp \left(-\frac{1}{4} \log \pi-\frac{1}{2} c\right)$.

So far, the main estimate (27) has been established as $z$ tends to 1 from the left, by real values. In fact, the formula (25) on which (27) rests holds for complex $t$ only constrained in such a way that $-\frac{\pi}{2}+\epsilon \leq \arg (t) \leq \frac{\pi}{2}-\epsilon$, for any $\epsilon>0$. The reason is that the converse mapping property (M4:ii) and in particular (25) rely on residues of the inverse Mellin integral that still converges when $t$ is restricted to such a wedge (cf. [6]). Thus, the expansion (27) actually holds true as $z \rightarrow 1$ in a sector, say,

$$
|\arg (1-z)|<\frac{\pi}{4} \text {. }
$$

Saddle Point Analysis. It remains to collect the information gathered on $S(z, 1)$ and recover $s_{n}=\left[z^{n}\right] S(z, 1)$ 
asymptotically. The inversion is provided by the Cauchy coefficient formula, that is,

$$
s_{n}=\frac{1}{2 \pi i} \oint \frac{S(z, 1)}{z^{n+1}} d z
$$

where the integration path is any simple loop around 0 inside the unit disk. The saddle point method [4], [9] summarized in Fig. 2 is now employed.

First, we provide a formula* for a standard set of functions that exhibit the same growth pattern as $S(z, 1)$ near $z=1$.

Lemma 4: For positive $A>0$, and reals $B$ and $C$, define $f(z)=f_{A, B, C}(z)$ as

$f(z)=\exp \left(\frac{A}{1-z}+B \log \frac{1}{1-z}+C \log \left(\frac{1}{z} \log \frac{1}{1-z}\right)\right)$.

Then, the $n$th Taylor coefficient of $f_{A, B, C}(z)$ satisfies asymptotically, for large $n$,

$$
\begin{aligned}
{\left[z^{n}\right] f_{A, B, C}(z)=2 } & \sqrt{A n}+\frac{1}{2}\left(B-\frac{3}{2}\right) \log n \\
& +C \log \log \sqrt{\frac{n}{A}} \\
& -\frac{1}{2} \log \left(4 \pi e^{-A} / \sqrt{A}\right)+o(1) .
\end{aligned}
$$

Proof: Problems of this kind have been considered by Wright [23] and others who, in particular, justify in detail that the saddle point method works in similar contexts. Therefore, we only outline the proof here. The starting point (see Fig. 2) is Cauchy's formula

$$
\left[z^{n}\right] f(z)=\frac{1}{2 \pi i} \oint e^{h(z)} d z
$$

where

$$
h(z)=\log f_{A, B, C}(z)-(n+1) \log z .
$$

In accordance with (S1) of Fig. 2, one chooses a saddle point contour that is a circle of radius $r$ defined by $h^{\prime}(r)=$ 0 . Asymptotically, one finds

$$
r=1-\sqrt{\frac{A}{n}}+\frac{B-A}{2 n}+o\left(n^{-1}\right),
$$

and

$$
\begin{array}{rl}
h(r)=2 & A \sqrt{\frac{n}{A}}+B \log \left(\sqrt{\frac{n}{A}}\right)+C \log \log \left(\sqrt{\frac{n}{A}}\right) \\
& +\frac{1}{2} A+o(1) .
\end{array}
$$

The "range" $\phi=\phi(n)$ of the saddle point, where most of the contribution of the contour integral is concentrated

\footnotetext{
${ }^{*}$ The computations here and in the rest of the section have been conducted with the help of the symbolic system Maple. Note that they require multiscale asymptotic manipulations for which the package based on the works of Salvy and Shackell [16] proved to be of special importance.
}

asymptotically, is dictated by the order of growth of derivatives; see $(\mathrm{S} 2)$. Here, $h^{\prime \prime}(r) \approx n^{3 / 2}$, while $h^{\prime \prime \prime}(r) \approx n^{2}$, so that

$$
\phi(n)=n^{-3 / 4} .
$$

In accordance with requirement (S3), tails are negligible since the function $\exp \left((1-z)^{-1}\right)$ decays very fast when going away from the real axis. In the central region, the local approximation (S4) applies, as seen by expansions near $z=1$. Thus requirements (S1), (S2), (S3), and (S4) are satisfied, implying, by (S5),

$$
\left[z^{n-1}\right] f(z)=\frac{1}{\sqrt{2 \pi\left|h^{\prime \prime}(r)\right|}} e^{h(r)}(1+o(1)) .
$$

Some simple algebra, using

$$
h^{\prime \prime}(r)=2 n \sqrt{n / A}(1+o(1)),
$$

yields the stated estimate (29).

Now, the function $S(z, 1)$ is only known to behave like $f(z)$ of Lemma 4 in the vicinity of 1 . In order to adapt the proof of Lemma 4 and legitimate the use of the resulting formula, we need to prove that $S(z, 1)$ decays fast away from the real axis.

Lemma 5 (Concentration property) Consider the ratio

$$
q(z)=\prod_{j=1}^{\infty}\left|\frac{\beta\left(z^{j}\right)}{\beta\left(|z|^{j}\right)}\right|
$$

Then, there exists a constant $c_{0}>0$ such that

$$
q\left(r e^{i \theta}\right)=O\left(e^{-c_{0}(1-r)^{-1}}\right)
$$

uniformly, for $\frac{1}{2} \leq r<1$ and $\left|\arg \left(r e^{i \theta}-1\right)\right|>\frac{\pi}{4}$.

Proof: In this proof the $c_{j}$ denote positive constants whose precise value is immaterial.

First, by the triangular inequality, a function like $\beta(z)$ that has nonnegative Taylor coefficients attains its maximum modulus on the positive real axis. More precisely, one has

$$
\sup _{\theta}\left|\beta\left(r e^{i \theta}\right)\right|=\beta(r) .
$$

Furthermore, by the converse triangular inequality, the maximum is uniquely attained on $|z|=r$ as soon as the function is aperiodic, which means the following: There is no $\widehat{\beta}(z)$ analytic at 0 such that $\beta(z)=z^{a} \widehat{\beta}\left(z^{b}\right)$ for integers $a, b$ and $b \geq 2$. This condition is obviously satisfied here since $\beta(z)=1+e^{-1} z+2\left(e^{-1} z\right)^{2}+\cdots$.

Fix some small angle parameter $\phi_{0}$, for instance, $\phi_{0}=$ $\frac{1}{10}$, and define

$$
\sigma(r)=\sup _{|\theta| \geq \phi_{0}}\left|\frac{\beta\left(r e^{i \theta}\right)}{\beta(r)}\right| .
$$

Then $\sigma(r)$ is continuous on the open interval $(0,1)$ where it satisfies $\sigma(r)<1$ while it tends to infinity when $r$ tends 
Input: A function $g(z)$ analytic in $|z|<R(0<R<+\infty)$ with nonnegative Taylor coefficients and "fast growth" as $z \rightarrow R^{-}$. Let $h(z):=\log g(z)-(n+1) \log z$.

Output: The asymptotic formula (35) for $g_{n}:=\left[z^{n}\right] g(z)$ derived from the Cauchy coefficient integral

$$
g_{n}=\frac{1}{2 i \pi} \int_{\gamma} g(z) \frac{d z}{z^{n+1}}=\frac{1}{2 i \pi} \int_{\gamma} e^{h(z)} d z
$$

where $\gamma$ is a loop around $z=0$.

(S1). SAddle Point Contour. Require that $g^{\prime}(z) / g(z) \rightarrow+\infty$ as $z \rightarrow R^{-}$. Let $r=r(n)$ be the unique positive root of the saddle point equation

$$
h^{\prime}(r)=0 \quad \text { or } \quad \frac{r g^{\prime}(r)}{g(r)}=n+1
$$

so that $r \rightarrow R$ as $n \rightarrow \infty$. The integral (30) is evaluated on $\gamma=\{z|| z \mid=r\}$.

(S2). BAsic SPLIT. Require that $h^{\prime \prime \prime}(r)^{1 / 3} h^{\prime \prime}(r)^{-1 / 2} \rightarrow 0$. Define $\phi=\phi(n)$ called the "range" of the saddle point by

$$
\phi=\left|h^{\prime \prime \prime}(r)^{-1 / 6} h^{\prime \prime}(r)^{-1 / 4}\right|
$$

so that $\phi \rightarrow 0, h^{\prime \prime}(r) \phi^{2} \rightarrow \infty$, and $h^{\prime \prime \prime}(r) \phi^{3} \rightarrow 0$. Split $\gamma=\gamma_{0} \cup \gamma_{1}$, where $\gamma_{0}=\{z \in \gamma|| \arg (z) \mid \leq \phi\}, \quad \gamma_{1}=$ $\{z \in \gamma|| \arg (z) \mid \geq \phi\}$.

(S3) Elimination of Tails. Require that $\left|g\left(r e^{i \theta}\right)\right| \leq\left|g\left(r e^{i \phi}\right)\right|$ on $\gamma_{1}$. Then, the tail integral satisfies the trivial bound,

$$
\left|\int_{\gamma_{1}} e^{h(z)} d z\right|=O\left(\left|e^{-h\left(r e^{i \phi}\right)}\right|\right) \text {. }
$$

(S4) Local approximation. Require that $h\left(r e^{i \theta}\right)-h(r)-\frac{1}{2} r^{2} \theta^{2} h^{\prime \prime}(r)=O\left(\left|h^{\prime \prime \prime}(r) \phi^{3}\right|\right)$ on $\gamma_{0}$. Then, the central integral is asymptotic to a complete Gaussian integral, and

$$
\frac{1}{2 i \pi} \int_{\gamma_{0}} e^{h(z)} d z=\frac{g(r) r^{-n}}{\sqrt{2 \pi h^{\prime \prime}(r)}}\left(1+O\left(\left|h^{\prime \prime \prime}(r) \phi^{3}\right|\right)\right)
$$

(S5) Collection. Requirements $(S 1),(S 2),(S 3),(S 4)$, imply the estimate:

$$
\left[z^{n}\right] g(z)=\frac{g(r) r^{-n}}{\sqrt{2 \pi h^{\prime \prime}(r)}}\left(1+O\left(\left|h^{\prime \prime \prime}(r) \phi^{3}\right|\right)\right) \sim \frac{g(r) r^{-n}}{\sqrt{2 \pi h^{\prime \prime}(r)}} .
$$

Fig. 2. The Saddle Point Algorithm.

to 1 . As a consequence, for each $\delta>0$, there exists an $A_{\delta}<1$ such that

$$
\sigma(r)<A_{\delta} \quad \text { for all } r \text { satisfying } \delta \leq r<1 .
$$

Consider the case where $z=r e^{i \theta}$ with $r \rightarrow 1$. Set $r=$ $e^{-\tau}$. The powers $z^{j}$ form a discrete set of points on a logarithmic spiral that winds about 0 . The number of such powers that have modulus larger than $\delta$ is

$$
\frac{\log \delta^{-1}}{\tau}+O(1)
$$

If $z=r e^{i \theta}$ and $|\theta| \geq \phi$, then a fraction of these points, namely,

$$
c_{1} \frac{\log A_{\delta}^{-1}}{\tau}+O(1)
$$

will lie outside of the region $\left|\arg \left(z^{j}\right)\right|<\phi$. Thus, by the bound (37), we find

$$
q\left(r e^{i \theta}\right)=O\left(e^{-c_{2} / \tau}\right)=O\left(e^{-c_{3} /(1-r)}\right) .
$$

This argument adapts when $z$ is close to the real axis as follows. It is assumed that $|\arg (z-1)|>\pi / 4$. Thus, $\arg (z)>c_{4} \theta$, for some $c_{4}>0$. Then, the winding number around 0 of the polygonal line with vertices the $z^{j}$ and $\left|z^{j}\right|>\delta$ is approximately

$$
\frac{1}{c_{4}} \log \delta^{-1} .
$$

In other words, fixing $\delta$ small enough ensures that at least one full winding takes place. In this case a number at least $c_{5} / \tau$ of the $z^{j}$ satisfying $\left|z^{j}\right|>A_{\delta}$ are such that $\left|\beta\left(z^{j}\right) / \beta\left(r^{j}\right)\right|<A_{\delta}$. Then, an estimate of type (38) holds,

$$
\left|q\left(r e^{i \theta}\right)\right|=O\left(e^{-c 6 / \tau}\right)=O\left(e^{-c_{7} /(1-r)}\right),
$$


albeit with different constants. The statement follows upon taking $c_{0}=\min \left(c_{3}, c_{7}\right)$.

We are now eventually ready to return to the estimate of $s_{n}$ in Lemma 2. In the region $|\arg (z-1)|<\frac{\pi}{4}$, the Mellin asymptotic estimates (25) and (27) apply. This shows that in this region,

$$
S(z, 1)=e^{o(1)} f_{A, B, C}(z) \quad(z \rightarrow 1),
$$

where the function $f$ is that of Lemma 4 and the constants $A, B, C$ have the values assigned by (27):

$$
A=c=\frac{\pi^{2}}{6}-1, \quad B=-\frac{1}{4}, \quad C=0 .
$$

In the complementary region, $|\arg (z-1)|>\frac{\pi}{4}$, the function $S(z, 1)$ is exponentially smaller than $(S(|z|, 1)$ by Lemma 5 . From these two facts, the saddle point estimates of Lemma 4 are seen to apply, by a trivial modification of the proof of that lemma. This concludes the proof of Equation (13) in Lemma 2.

Moments. It remains to complete the evaluation of $\mu_{n}$ and $\sigma_{n}^{2}$, following the same principles as before. Start with $\mu_{n}=\mathbf{E}\left[K_{n}\right]$, with the goal of establishing the evaluation (14) of Lemma 2 . It is necessary to estimate $\left[z^{n}\right] S_{u}^{\prime}(z, 1)$, with

$$
S_{u}^{\prime}(z, 1)=S(z, 1) \sum_{k=0}^{\infty} z^{k} \frac{\beta^{\prime}\left(z^{k}\right)}{\beta\left(z^{k}\right)} \text {. }
$$

Let

$$
D_{1}(z)=\sum_{k=0}^{\infty} \alpha\left(z^{k}\right), \quad \text { where } \quad \alpha(z)=z \frac{\beta^{\prime}(z)}{\beta(z)} .
$$

Via the substitution $z=e^{-t}$, the function $D_{1}\left(e^{-t}\right)$ falls under the harmonic sum property (M3) of Fig 1, so that its Mellin transform is

$$
\mathcal{M}\left(D_{1}\left(e^{-t}\right) ; s\right)=\zeta(s) \mathcal{M}\left(\alpha\left(e^{-t}\right) ; s\right) .
$$

The asymptotic expansion

$$
\alpha\left(e^{-t}\right)=\frac{1}{2 t}-\frac{\sqrt{2}}{6} \frac{1}{\sqrt{t}}-\frac{1}{18}+O(\sqrt{t}),
$$

gives the singular expansion of the corresponding Mellin transform, by (M4:i). This in turn yields yields the singular expansion of $\mathcal{M}\left(D_{1}\left(e^{-t}\right) ; s\right)$. Then, the converse mapping property (M4:ii) gives back $D\left(e^{-t}\right)$ at $t \sim 0$, hence,

$$
\begin{aligned}
D_{1}(z)= & \frac{1}{2} \frac{1}{1-z} \log \frac{1}{1-z}+\frac{1}{2} \frac{\gamma}{1-z} \\
& -\frac{1}{6} \frac{\sqrt{2} \zeta\left(\frac{1}{2}\right)}{\sqrt{1-z}}-\frac{1}{4} \log \frac{1}{1-z}+O(1),
\end{aligned}
$$

where $\gamma=0.577 \ldots$ is the Euler constant. The combination of this last estimate and the main asymptotic form of $S(z, 1)$ in $(27)$ yields

$S_{u}^{\prime}(z, 1) \underset{z \rightarrow 1}{\sim} \frac{1}{2} a \exp \left(\frac{c}{1-z}+\frac{3}{4} \log \frac{1}{1-z}+\log \log \frac{1}{1-z}\right)$, where $a$ is the same constant as in (27). Like for $S(z, 1)$, the derivative $S_{u}^{\prime}(z, 1)$ is amenable to Lemma 4 , and this proves the asymptotic form of $\mu_{n}$, as stated in (14) of Lemma 2.

Finally, we need to justify (15) that represents a bound on the variance of $K_{n}$. The computations follow the same steps as above, so we only sketch them briefly. One needs to estimate a second derivative,

$$
\frac{S_{u u}^{\prime \prime}(z, 1)}{S(z, 1)}=D_{2}(z)+D_{1}^{2}(z)
$$

where

$$
D_{2}(z)=\sum_{k=0}^{\infty} z^{2 k} \frac{\beta^{\prime \prime}\left(z^{k}\right)}{\beta\left(z^{k}\right)}-\left(\frac{z^{k} \beta^{\prime}\left(z^{k}\right)}{\beta\left(z^{k}\right)}\right)^{2} .
$$

The sum above is again a harmonic sum that is amenable to Mellin analysis, with the result that

$$
D_{2}(z)=\frac{\zeta(2)}{2} \frac{1}{(1-z)^{2}}+O\left((1-z)^{-3 / 2}\right) .
$$

Then we appeal again to Lemma 4 to achieve the transfer to coefficients. Somewhat tedious calculations (that were assisted by the computer algebra system MAPLE) show that the leading term in $n \log ^{2} n$ of the second moment cancels with the square of the mean $\mu_{n}$. Hence, the variance cannot be larger than than $O(n \log n)$. This establishes the second moment estimate (15) of Lemma 2 and hence it completes the proof of Theorem 1.

\section{Numerical Estimates}

Numerical verifications support extremely well the claims made in the introduction about the accuracy of asymptotic expansions based on analytic methods, even when the values of $n$ are far from the asymptotic regime. Our main result states that the function

$$
\varphi(n)=\frac{2}{\log 2} \sqrt{\left(\frac{\pi^{2}}{6}-1\right) n}-\frac{5}{8} \log _{2} n+\frac{1}{2} \log _{2} \log n+K,
$$

where

$$
K=\frac{1}{8} \lg \left(\frac{\pi^{2} / 6-1}{2^{12} \pi^{2}}\right) \approx-1.99197,
$$

is such that $R_{n}=\varphi(n)+o(1)$. In fact observation of the values of the difference $\delta(n)=R_{n}-\varphi(n)$, for which a sample is given by the following table,

\begin{tabular}{lcccccc}
\hline$n:$ & 3 & 5 & 10 & 20 & 50 & 100 \\
\hline$\delta(n):$ & 0.223 & 0.026 & 0.128 & 0.055 & 0.002 & -0.010 \\
\hline
\end{tabular}

shows that the quantity $\varphi(n)+1 / 2$ estimates the redundancy $R_{n}^{*}\left(\mathcal{R}_{0}\right)$ of Theorem 1 within at most 1 , for all values of $n \geq 3$.

Acknowledgements. We thank Yiannis Kontoyiannis (Purdue University) for pointing to us the paper of Csiszár and Shields. The second author is also grateful Gadiel Seroussi (HPL, Palo Alto) and Marcelo Weinberger (HPL, Palo Alto) for a very enjoyable and productive stay at Hewlett-Packart Labs at Palo Alto in summer 1997, where this work was initiated. Thanks finally to Bruno Salvy (INRIA, France) for assistance with symbolic computations. 
[1] G. E. Andrews, The Theory of Partitions, Cambridge University Press, 1984.

[2] R. Corless, G. Gonnet, D. Hare, D. Jeffrey and D. Knuth, On the Lambert W Function, Adv. Computational Mathematics, 5, 329-359, 1996.

[3] I. Csiszár and P. Shields, Redundancy Rates for Renewal and Other Processes, IEEE Trans. Information Theory, 42, 2065$2072,1996$.

[4] N. G. De Bruijn, Asymptotic Methods in Analysis. Dover, 1981.

[5] P. Flajolet and A. Odlyzko, Singularity Analysis of Generating Functions, SIAM J. Disc. Methods, 3, 216-240, 1990.

[6] P. Flajolet, X. Gourdon, P. Dumas, Mellin Transforms and Asymptotics: Harmonic Sums, Theoretical Computer Science, 144, 3-58, 1995.

[7] P. Flajolet and R. Sedgewick, Analytic Combinatorics, book in preparation; preliminary version available on http://pauillac.inria.fr/algo/flajolet/.

[8] G.H. Hardy and S. Ramanujan, Asymptotic Formulæ in Combinatorial Analysis, Proc. London Math. Soc., Ser. 2, XVII, 75-115, 1918.

[9] P. Henrici, Applied and Computational Complex Analysis, 3 vol. John Wiley, 1977.

[10] P. Jacquet and W. Szpankowski, Asymptotic Behavior of the Lempel-Ziv Parsing Scheme and Digital Search Trees, Theoretical Computer Science, 144, 161-197, 1995.

[11] P. Jacquet and W. Szpankowski, Entropy Computations Via Analytic Depoissonization, IEEE Trans. Information Theory, to appear.

[12] P. Jacquet and W. Szpankowski, A verage Profile of The LempelZiv Parsing Scheme For a Markovian Source, Purdue University, CDS-TR-98-029, 1998.

[13] G. Louchard and W. Szpankowski, On the Average Redundancy Rate of the Lempel-Ziv Code, IEEE Trans. Information Theory, 43, 2-8, 1997.

[14] A. Odlyzko, Asymptotic Enumeration, in Handbook of Combinatorics, Vol. II, (Eds. R. Graham, M. Götschel and L. Lovász), Elsevier Science, 1063-1229, 1995.

[15] J. Rissanen, Complexity of Strings in the Class of Markov Sources, IEEE Trans. Information Theory, 30, 526-532, 1984.

[16] B. Salvy and J. Shackell, Symbolic Asymptotics: Multiseries of Inverse Functions, INRIA-TR-3264, 1997.

[17] S. Savari, Redundancy of the Lempel-Ziv Incremental Parsing Rule, IEEE Trans. Information Theory, 43, 9-21 (1997).

[18] P. Shields, Universal Redundancy Rates Do Not Exist, IEEE Trans. Information Theory, 39, 520-524, 1993.

[19] Y. Shtarkov, Universal Sequential Coding of Single Messages, Problems of Information Transmission, 23, 175-186, 1987.

[20] W. Szpankowski, On Asymptotics of Certain Recurrences Arising in Universal Coding, Problems of Information Transmission, 34, No.2, 55-61, 1998.

[21] W. Szpankowski, Average Case Analysis of Algorithms, in Handbook of Algorithms and Theory of Computation (Ed. M. Atallah), CRC 1998.

[22] M. Weinberger, N. Merhav, and M. Feder, Optimal Sequential Probability Assignments for Individual Sequences, IEEE Trans. Information Theory, 40, 384-396, 1994.

[23] E. M. Wright, The Coefficients of Certain Power Series, The J. of the London Mathematical Society, 7, 256-262, 1932.

[24] A. J. Wyner, The Redundancy and Distribution of the Phrase Lengths of the Fixed-Database Lempel-Ziv Algorithm, IEEE Trans. Information Theory, 43, 1439-1465, 1997.

[25] J. Ziv, Back from Infinity: A Constrained Resources Approach to Information Theory, IEEE Information Theory Society Newsletter, 48, 30-33, 1998. 


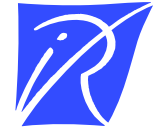

Unité de recherche INRIA Lorraine, Technopôle de Nancy-Brabois, Campus scientifique, 615 rue du Jardin Botanique, BP 101, 54600 VILLERS LÈS NANCY

Unité de recherche INRIA Rennes, Irisa, Campus universitaire de Beaulieu, 35042 RENNES Cedex Unité de recherche INRIA Rhône-Alpes, 655, avenue de l'Europe, 38330 MONTBONNOT ST MARTIN

Unité de recherche INRIA Rocquencourt, Domaine de Voluceau, Rocquencourt, BP 105, 78153 LE CHESNAY Cedex

Unité de recherche INRIA Sophia-Antipolis, 2004 route des Lucioles, BP 93, 06902 SOPHIA-ANTIPOLIS Cedex

Éditeur

INRIA, Domaine de Voluceau, Rocquencourt, BP 105, 78153 LE CHESNAY Cedex

(France)

http://www.inria.fr

ISSN 0249-6399 